Sharif University of Technology
Scientia Iranica
SCIENTIA
I RAN ICA
http://scientiairanica.sharif.edu

\title{
Analysis of the interaction between destination and departure time choices
}

\author{
A. Rasaizadi and S. Seyedabrishami* \\ Department of Civil and Environmental Engineering, Tarbiat Modares University, Tehran, P.O. Box 14115-111, Iran.
}

Received 10 October 2019; received in revised form 5 August 2020; accepted 3 May 2021

\author{
KEYWORDS \\ Joint modeling; \\ Copula; \\ Destination choice; \\ Departure time \\ choice; \\ Multinomial logit.
}

\begin{abstract}
Departure time and destination choices are interrelated decisions that affect the demand estimation for urban transportation. A majority of the previous studies have ignored this interrelation and regarded these decisions as independent. On the contrary, some other studies have utilized a hierarchy structure; however, the literature suggested that destination and departure time should be simultaneously selected before the commencing of trips. The present study employed a joint model using the copula functions to explore the interdependency between the destination and departure time choices. The destination choice modeling was developed using a multinomial logit model, and a binary logit model was used for modeling the departure time choice. To obtain a better fitted joint model, some copulas were utilized and then, the frank copula was selected for the final model. The obtained results indicated that there were some common unobserved factors between these decisions by estimating the copula dependence parameters with high statistical significance. Furthermore, there were some commonly observed factors such as socio-demographic and travel characteristics that appeared in the utility functions of both models.
\end{abstract}

(C) 2021 Sharif University of Technology. All rights reserved.

\section{Introduction}

Generally, traffic congestion at peak hours is caused by significant travel demand for some specific destinations (for example, administrative destination) at particular hours. Congestion will consequently cause a number of problems such as air pollution, noise pollution, loss of energy, and safety reduction. With a rapid growth of urbanization, expanding the capacity of the transport network is no longer an efficient and economical option. Managing travel demand is an alternative to tackle

\footnotetext{
*. Corresponding author. Tel.: +982182884914;

Fax: +982182884964

E-mail addresses: arash_rasaizadi@modares.ac.ir (A.

Rasaizadi); seyedabrishami@modares.ac.ir ( $S$.

Seyedabrishami)
}

doi: 10.24200 /sci. 2021.54500 .3779 congestion in large metropolitan areas with a limited infrastructure capacity.

Keeping in mind the significant impact of destination and departure time choices on the traffic load on the network, one can feel the necessity to understand how to efficiently model these decisions. In this respect, it is vital to find a suitable modeling structure that can depict travelers' behavior towards destination choice according to the land use and departure time choice [1].

According to the literature, destination and departure time choices are interrelated choices. The selected destination has a significant effect on when trips began and how the selected departure time could affect where trips end. For example, if an individual decides to take a shopping trip, choosing the destination with business land use and peak hour is more expected than choosing other destinations and off-peak (early and late) hours. Usually, people choose the departure time and destination simultaneously before making their 
trips. The interaction between these two decisions can be systematically captured by including variables relating to them. There might also be some unobserved correlations between the two decisions that can be investigated in an appropriate modeling formulation. This study explores the application of copula functions to capture the correlation between destination and departure time choices.

\section{Literature review}

A majority of studies have ignored the relationship between destination and departure times. For example, Thorhauge et al. [2] employed discrete choice models, such as a multinomial logit model, to analyze the departure time for work trips without considering the reciprocal effect of destination. Sultana [3] also studied the departure time choice for non-work trips. Sasic and Habib [4] investigated the modeling departure time choice using the heteroskedastic generalized model for home-based commuting trips. Furthermore, several studies have focused on destination choice without considering the effect departure time $[5,6]$. For example, Clifton et al. [7] employed the multinomial logit model to study the destination choice for pedestrian travel.

Some other studies analyzed the interaction of departure time or destination choices with other travel decisions $[8,9]$. Ding et al. [10] employed a crossnested logit to model the trip mode and departure time for urban commuting trips. Bhat [11] utilized the multinomial and nested logits to analyze the mode and departure time of shopping trips. Moreover, Elmorssy and Onur [12] used the generalized nested logit to study the interaction among the departure time, destination choice, and travel mode for non-mandatory trips. Some studies have considered the interaction among discrete travel decisions using a copula-based joint model [13,14]. For example, Ermagun et al. [15] investigated the joint modeling of parent escort decision and student mode choice for educational trips and pointed out the benefits of using the Copula-based structure. Rasaizadi and Kermanshah [13] examined the endogenous correlation among the number of stops and trip mode using the Copula-based model.

To the best of the author's knowledge, no study has been found that could jointly model the departure time and destination. This study employed a copulabased framework to explore the interdependency between departure time and destination choices. This framework is not characterized by any hierarchy among decisions like other models, such as the nested logit model, with a hierarchy structure. While the destination modeling was developed using the multinomial logit model, departure time choice was modeled using the binary logit model. Seyedabrishami and Izadi [1] and Bhat and Sener [16] pointed to the benefits of using the copula-based framework in the following:

1. It is a powerful technique to accommodate spatial error correlation;

2. It does not impose any limiting distribution assumption about the type of dependency;

3. It leads to a closed-form without intensive computational;

4. It is easy to apply through a standard and direct maximum likelihood inference procedure.

The present study employed the copula-based joint modeling structure to model the departure time and destination, utilized the travel dataset of Qazvin, Iran as a developing country, and evaluated the aggregate elasticities.

In this study, the joint model of destination and departure time choice was developed for non-work home-based trips. Work trips (education trips are also considered as work trips) are excluded. The passenger has a limited choice on the departure time. Departure time on trips starting from home is more flexible.

\section{Methodology}

This section presents a detailed discussion about the formulation of the joint structure for destination and time of day choice behavior.

\subsection{Destination model structure}

The multinomial logit structure was employed to model the destination choice. The choice set comprised non-residential, residential-educational, residentialadministrative, and residential-business zones. Here, " $q$ " is the index of a person, " $i$ " represents the destination, and " $h_{q i}$ " denotes the utility of destination " $i$ " of person " $q$ ". In such a case, we can define the utility function as $[1,17]$ :

$$
h_{q i}=\beta x_{q i}+\varepsilon_{q i},
$$

where $x_{q i}$ is the vector of exogenous variables, $\beta$ the coefficient vector of independent variables that must be estimated, and $\varepsilon_{q i}$ the error term (random term) of $h_{q i}$. Assume that $\varepsilon_{q i}$ follows the independent and identically distribution (iid), whose location and scale parameters are equal to 0 and 1, respectively [1]. Based on the utility theory, " $q$ " selects the option " $i$ ", the utility of which is more significant than the maximum utility of the other destinations for " $q$ ". This term can be written as follows [17]:

$$
h_{q i}>\max _{j \neq i} h_{q j}
$$

The dependent variable is called $r_{q i}$ which is a binary variable taking the value of one if the destination " $i$ " 
is chosen by " $q$ "; otherwise, it would be zero [18]. Further, $v_{q i}$ can be defined as $[1,17]$ :

$$
v_{q i}=\left\{\max _{j \neq i} h_{q j}\right\}-\varepsilon_{q i} .
$$

By combining Eq. (1) and Inequality (2), we derive the following inequality:

$$
\beta x_{q i}+\varepsilon_{q i}>\max _{j \neq i} h_{q j},
$$

which can be re-written by substituting $v_{q i}$ from Eq. (3) into Inequality (4) [1]:

$$
\beta x_{q i}>v_{q i} \text {. }
$$

So, $r_{q i}=1$ if and only if $\beta x_{q i}>v_{q i}$.

Based on Eq. (3), $v_{q i}$ is obtained from the differentiation of the utility of the other destination and error term of the chosen destination. Since both of them follow the iid extreme value distribution, the random variable of $v_{q i}$ follows the logistic distribution obtained from the differentiation of iid distributed variables [1]. The marginal distribution of $v_{q i}$ presented in Eq. (6) is [17]:

$$
F_{i}\left(\beta x_{q i}\right)=\operatorname{Pr}\left(v_{q i}<\beta x_{q i}\right)=\frac{\exp \left(\beta x_{q i}\right)}{\sum_{j} \exp \left(\beta x_{q j}\right)} .
$$

\subsection{Departure time choice model structure}

The binomial logit is used for modeling the departure time choice. The choice set consists of two options: traffic off-peak hours and traffic peak hours. Here, " $q$ ", " $k$ ", and " $u_{q k}$ " represent a person, departure time, and utility of choosing " $k$ " for person " $q$ ", respectively. We have $[1,17]$ :

$$
u_{q k}=\gamma z_{q k}+\mu_{q k}
$$

where $z_{q k}$ is the vector of exogenous variables, $\gamma$ a vector of parameters that must be estimated, and $\mu_{q k}$ the random error term of the utility function. In addition, $\mu_{q k}$ follows the independent and identically distribution (iid), whose location and scale parameters are equal to 0 and $1[1,17]$. Moreover, " $q$ " chooses " $k$ " if $u_{q k}$ is more than other utilities.

$$
u_{q k}>u_{q l}(l \neq k) \text {. }
$$

So we have [1]:

$$
\begin{aligned}
& \gamma z_{q k}+\mu_{q k}>\mu_{q l}, \\
& \mu_{q k}-\mu_{q l}>-\gamma z_{q k} .
\end{aligned}
$$

Moreover, $\tau_{q k l}$ is defined as $\mu_{q k}-\mu_{q l}=\tau_{q k l}$; then, we have $[1,17]$ :

$$
\tau_{q k l}>-\gamma z_{q k}
$$

So, $S_{q K}=1$ if and only if $\tau_{q k l}>-\gamma z_{q k}$.

The random variable of $\tau_{q k l}$ which is derived from the differentiation of $\mu_{q k}$ and $\mu_{q l}$ follows the logistic distribution. Then, the marginal distribution of $\tau_{q k l}$ presented in Eq. (12) can be written as follows [1,17]:

$$
G_{k}\left(-\gamma z_{q k}\right)=\frac{\exp \left(-\gamma z_{q k}\right)}{1+\exp \left(-\gamma z_{q k}\right)}
$$

\subsection{Joint model structure}

The probability where " $q$ " selects destination " $i$ " and departure time " $k$ " is [1]:

$$
\begin{aligned}
\operatorname{Pr}\left[r_{q i}\right. & \left.=1, s_{q k}=1\right]=\operatorname{Pr}\left[v_{q i}\left\langle\beta x_{q i}, \tau_{q k l}\right\rangle-\gamma z_{q k}\right] \\
& =\operatorname{Pr}\left[v_{q i}<\beta x_{q i}\right]-\operatorname{Pr}\left[v_{q i}<\beta x_{q i} \tau_{q k l}<-\gamma z_{q k}\right] .
\end{aligned}
$$

The probability function should calculate a bivariate distribution among the random terms of models. The copula comprises cumulative distribution functions which consist of the marginal densities $[1,18,19]$. Eq. (13) can be rewritten as follows:

$$
\begin{aligned}
\operatorname{Pr}\left[r_{q i}\right. & \left.=1, s_{q k}=1\right]=u_{q i}-C_{\theta_{i k}}\left(u_{q i}, u_{q k}\right) \\
& =F_{i}\left(\beta x_{q i}\right)-C_{\theta_{i k}}\left(F_{i}\left(\beta x_{q i}\right), G_{k}\left(-\gamma z_{q k}\right)\right],
\end{aligned}
$$

where $u_{q i}=F_{i}\left(\beta x_{q i}\right), u_{q k}=G_{i}\left(-\gamma z_{q k}\right) . F$ and $G$ are marginal densities of destination and departure time choices. Here, $\theta_{i k}$ is the dependence parameter of copula that represents the correlation between random terms of option " $i$ " and option " $k$ " $[1,18,19]$. In this study, Frank and AMH (Ali-Mikhail-Haq copula) copulas were used that could estimate the dependence parameter. Moreover, both positive and negative correlations (dependence parameters) were taken into account. These two copula functions were selected because they could explore both positive and negative dependencies among the error terms. Since the copula dependence parameter of other copula functions such as Clayton, Joe, and Gumbel was limited to positive values or excluded zero value, they could not depict negative dependencies or independency; therefore, they were not employed in this study. The product copula shows independent random terms. Table 1 introduces used copulas [1].

Table 1. Attributes of copulas [1].

\begin{tabular}{lcc}
\hline Copula & $C\left(u_{1}, u_{2}\right)$ & Range of $\theta$ \\
Product & $u_{1} u_{2}$ & - \\
Frank & $-\theta^{-1} \log \left\{1+\frac{\left(e^{-\theta u_{1}}-1\right)\left(e^{-\theta u_{2}}-1\right)}{e^{-\theta}-1}\right\}$ & $(-\infty, \infty)$ \\
AMH & $u_{1} u_{2}\left(1-\theta\left(1-u_{1}\right)\left(1-u_{2}\right)\right)^{-1}$ & {$[-1,1]$} \\
\hline
\end{tabular}


The implication of copulas is not limited to the joint modeling of different choices. For example, Liu et al. [20] proposed a correlation and risk measurement model using Markov-switching mixed-Clayton copula.

\subsection{Estimation process}

Define a binary variable $M_{q i k}$ such that if person " $q$ " selects the destination " $i$ " and departure time " $k$ ", $M_{q i k}$ for that person equals one and for the other destinations, it equals 0 .

The likelihood function of this model is as follows [18]:

$$
\log L=\sum_{q=1}^{Q}\left(\sum_{i=1}^{I} \sum_{k=1}^{K} M_{q i k} \log \left[\operatorname{prob}\left(r_{q i}, s_{q k}\right)\right]\right)
$$

where $Q$ is the number of individuals, $I$ the number of destination alternatives, and $K$ the number of departure time choices.

By maximizing this function, the parameters of these models including $\beta, \gamma$, and $\theta$ can be estimated. In order to maximize this function, the code developed in R-studio is used [1].

\section{Database}

The travel data of Qazvin, Iran conducted in 2010 were taken into consideration in this study. The number of non-work trips is 6135 trips.

The departure land-use model choice set includes non-residential, residential-educational, residentialadministrative, and residential-business. The choice set of departure time consists of peak and off-peak hours. Regarding the frequency of trips during a day, 6 AM-8 AM, 12 PM-13 PM, and 17 PM-18 PM were considered as peak hours. Table 2 shows the frequency of choice sets and Table 3 defines independent variables in the dataset [1].

\section{Model estimation, results, and discussion}

For the departure time model, the base is set to the off-peak hour option whose utility equals zero, and

Table 2. Frequency of models choice set.

\begin{tabular}{lcc}
\hline \multicolumn{1}{c}{ Choices } & Frequency & Share \\
\hline None-residential & 1691 & 0.276 \\
Residential-educational & 893 & 0.146 \\
Residential-administrative & 1273 & 0.207 \\
Residential-business & 2278 & 0.371 \\
\hline Peak hours & 1700 & 0.277 \\
Off-peak hours & 4435 & 0.723 \\
\hline
\end{tabular}

the coefficients of another option (peak hours) are estimated relative to the base option.

Copula functions offer different descriptions about the interaction among random terms of models. The highest log-likelihood is achieved by the frank copula and the detailed results of this model are presented. In addition, the higher log-likelihood of Frank copula compared to the product copula was indicative of the significant role of the interaction [1]. The proposed model is also calibrated with parameterization strategies, and the dependency parameter is allowed to vary across observations [21]. Since the parameterized model does not increase log-likelihood significantly, the unparameterized model is analyzed in the following. Table 4 compares the performances of different calibrated models. Table 5 shows the results achieved through frank copula.

\section{Analysis of results}

The $t$ statistics estimated for the copula dependence parameters showed the correlation between the error terms of traffic peak hours and destination. While these parameters have a weak significance level for the non-residential and residential-administrative destinations, they have a strong significance level for the residential-educational and residential-business destinations. In other words, decisions about destination and departure time are not much correlated for the non-residential and residential-administrative areas; however, there is a strong interaction between the peak hours and residential-educational and residentialbusiness destinations. Regarding the estimated sign for copula dependence parameters, several correlated unobserved factors have the same effects on selecting the destination and peak hours [9]. For example, the negative estimated copula dependence parameter for residential-business destinations indicates that there are some unobserved influential factors that have different effects on choosing residential-business and peak hours. These factors are commonly related to the personality of travelers. For example, a person who does not like crowded places and congested traffic does not choose the residential-business destination (usually crowed at traffic peak hours) and peak hour simultaneously. Therefore, the sign of copula dependence parameter for residential-business seems to be logical based on this unobserved factor.

Regarding the estimated coefficient for independent variables, it can be said that res-bus is a more favorable destination for individuals over 31 years old than that for other individuals. Also, the resadm destination has the least favorability for individuals between 5-30 years old. Usually, this age bracket is not employed and does not need to go to administrative destinations [1]. Individuals between 
Table 3. Independent variables [1].

\begin{tabular}{|c|c|c|c|}
\hline Information & Variables & Definitions & Shares \\
\hline \multirow{4}{*}{ Age } & AGE $5-18$ & 1: if age is between 6 and $18 ; 0$ : otherwise & 10.84 \\
\hline & AGE 19-30 & 1: if age is between 19 and $30 ; 0$ : otherwise & 22.94 \\
\hline & AGE $31-41$ & 1: if age is between 31 and $41 ; 0$ : otherwise & 30.99 \\
\hline & $\mathrm{AGE}>41$ & 1: if age is more than $41 ; 0$ : otherwise & 25.24 \\
\hline Sex & SEX & 1: for male; 0 : otherwise & 38.30 \\
\hline \multirow{4}{*}{ Job } & ADM.JOB & 1: for administrative job, 0 : otherwise & 12.42 \\
\hline & SERV.JOB & 1: for service job, 0 : otherwise & 13.40 \\
\hline & EDU.JOB & 1: for educational job, 0 : otherwise & 14.42 \\
\hline & Other & 1: for other jobs, 0 : otherwise & 59.76 \\
\hline \multirow{3}{*}{ Education } & LOW.EDU & 1: for high school education, 0 : otherwise & 49.45 \\
\hline & MED.EDU & 1: for associate's or bachelor's degree, 0 : otherwise & 37.70 \\
\hline & HIGH.EDU & 1: for master's degree or doctorate, 0 : otherwise & 12.87 \\
\hline Driving license & DL & 1: for having a driving license, 0 : otherwise & 48.81 \\
\hline Travel distance & LGH & Travel distance $(\mathrm{km})$ & 2.88 \\
\hline Travel time & $\mathrm{TT}$ & Travel time (min) & 6.73 \\
\hline Walking time & WT & Walking time to the bus station (min) & 7.5 \\
\hline \multirow{4}{*}{ Trip aim } & Shopping & 1: for shopping aim, 0: otherwise & 36.84 \\
\hline & Recreational & 1: for recreation aim, 0 : otherwise & 16.67 \\
\hline & Visiting & 1: for visiting aim, 0 : otherwise & 26.39 \\
\hline & Other & 1: for other aims aim, 0: otherwise & 20.10 \\
\hline \multirow{4}{*}{ Trip mode } & Private & 1: for private vehicle, 0 : otherwise & 0.24 \\
\hline & Taxi & 1: for taxi, 0 : otherwise & 0.31 \\
\hline & Bus & 1: for bus, 0 : otherwise & 0.15 \\
\hline & Active & 1: for active transport, 0 : otherwise & 0.30 \\
\hline
\end{tabular}

Table 4. Models' performance comparison.

\begin{tabular}{cc}
\hline Model & Log-likelihood \\
\hline Frank copula & -10937.9 \\
AMH copula & -10941 \\
Product copula & -10967.5 \\
\hline
\end{tabular}

19-30 have greater tendencies to select peak hours, res-bus, and non-res destination. The probability of choosing a non-res destination is high for individuals over 41 years old and low for individuals between 518 years old. The non-res destination has greater favorability for administrative employment than that for other individuals. The probability of choosing peak hours is low for administrative employees and students. These individuals have their mandatory trips to school and work at peak hours; therefore, there is no more time to do non-mandatory purposes at peak hours [22]. Individuals with low and medium levels of education have more tendency for choosing res-bus than for individuals with a high level of education. Favorability of res-adm is low for individuals with medium and high levels of education. Regarding the tight time budget of individuals with a high level of education, its negative coefficient in peak hour utility function can be expected as the positive coefficient for the low level of education [13]. The increase in 
Table 5. Model results using frank copula.

\begin{tabular}{|c|c|c|c|c|c|c|}
\hline \multirow[b]{2}{*}{ Variable } & \multicolumn{4}{|c|}{ MNL } & \multicolumn{2}{|r|}{ BL } \\
\hline & Non-residential & $\begin{array}{l}\text { Residential- } \\
\text { educational }\end{array}$ & $\begin{array}{c}\text { Residential- } \\
\text { administrative }\end{array}$ & $\begin{array}{c}\text { Residential- } \\
\text { business }\end{array}$ & $\begin{array}{c}\text { Off-peak } \\
\text { hours }\end{array}$ & Peak hours \\
\hline $\begin{array}{l}\text { Copula dependence } \\
\text { parameter }\end{array}$ & $0.484(0.869)$ & $-1.095(-2.001)$ & $-0.297(-0.578)$ & $-0.768(-1.394)$ & - & - \\
\hline Constants & - & $0.862(4.656)$ & $0.959(4.916)$ & $0.840(4.563)$ & - & $0.653(3.227)$ \\
\hline
\end{tabular}

Personal info.

AGE

$\begin{array}{lccccc}\text { AGE 5-18 } & -0.467(-2.542) & - & -0.320(-2.748) & - & - \\ \text { AGE 19-30 } & - & -0.781(-6.712) & -0.407(-4.037) & - & - \\ \text { AGE 31-41 } & - & - & - & 0.350(4.241) & - \\ \text { AGE }>\text { 41 } & 0.306(3.674) & - & - & 0.488(5.323) & -\end{array}$

JOB

\begin{tabular}{|c|c|c|c|c|c|c|}
\hline ADM.JOB & - & - & - & - & - & $-0.247(-2.340)$ \\
\hline SERV.JOB & $-0.258(-1.873)$ & $-0.223(-1.869)$ & - & - & 一 & - \\
\hline EDU.JOB & $-0.513(-2.997)$ & - & - & - & - & $-0.319(-3.801)$ \\
\hline Other & $-0.278(-2.426)$ & - & - & - & - & - \\
\hline
\end{tabular}

\section{Education}

LOW.EDU

$0.436(4.383)$
-
-

$$
-0.183(-1.873)
$$

$0.252(2.146)$

- $0.380(5.580)$

MED.EDU

HIGH.EDU

$0.249(2.419)$

$-0.389(-2.874)$

$-0.172(-1.646)$

Travel info.

TT

LGH

WT

$$
\begin{array}{cc}
-0.040(-1.288) & -0.269(-6.377) \\
- & 0.278(3.418) \\
0.069(7.882) & -
\end{array}
$$

$\frac{-}{-0.233(-3.178)}$

$$
0.183(5.434)
$$$$
-0.610(-9.411)
$$$$
-0.024(-2.967)
$$

\section{AIM}

Shopping

Recreational

Visiting

Other

$$
\begin{gathered}
0.678(6.110) \\
- \\
-0.322(-2.640)
\end{gathered}
$$$$
-0
$$$$
-0.238(-1.746)
$$$$
-
$$$$
0.191 \text { (1.630) }
$$$$
0.884(5.390)
$$$$
0.563(3.995)
$$

$$
0.173(1.859)
$$

\begin{tabular}{|c|c|c|c|c|c|c|}
\hline Personal vehicle & $-0.444(-5.079)$ & - & - & - & - & $-0.353(-4.422)$ \\
\hline Taxi & - & - & - & - & - & - \\
\hline Bus & - & $-0.302(-2.415)$ & - & $-0.219(-2.468)$ & - & - \\
\hline Active transport & - & - & $0.299(3.298)$ & $0.197(2.415)$ & - & $-0.674(-9.193)$ \\
\hline
\end{tabular}$$
\text { - } \quad 0.887(10.951)
$$$$
-0.341(-3.060)
$$$$
0.501(5.146)
$$$$
\text { - }
$$

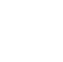

\section{Mode}


Table 6. Elasticity for final model.

\begin{tabular}{lcccccccc}
\hline Variable & $\begin{array}{c}\text { Non-res } \\
\text { and } \\
\text { off-peak }\end{array}$ & $\begin{array}{c}\text { Res-edu } \\
\text { and } \\
\text { off-peak }\end{array}$ & $\begin{array}{c}\text { Res-adm } \\
\text { and } \\
\text { off-peak }\end{array}$ & $\begin{array}{c}\text { Res-bus } \\
\text { and } \\
\text { off-peak }\end{array}$ & $\begin{array}{c}\text { Non-res } \\
\text { and peak }\end{array}$ & $\begin{array}{c}\text { Res-edu } \\
\text { and peak }\end{array}$ & $\begin{array}{c}\text { Res-adm } \\
\text { and peak }\end{array}$ & $\begin{array}{c}\text { Res-bus } \\
\text { and peak }\end{array}$ \\
\hline TT & -0.41 & -2.03 & -0.14 & 1.18 & -0.44 & -1.91 & -0.14 & 1.04 \\
WT & 0.42 & -0.12 & -0.12 & -0.31 & 0.45 & -0.11 & -0.11 & -0.28 \\
LGH & 0.63 & 1.51 & -0.05 & -1.20 & 0.68 & 1.42 & -0.05 & -1.06 \\
\hline
\end{tabular}

travel time has a negative effect on choosing non-res and res-edu destinations, and the increase in travel distance has a negative effect on choosing res-adm and res-bus destinations. Increasing walking time to the bus station decreases res-bus favorability and increases non-res favorability. According to the destination, it can be said that non-res enjoys more favorability for a shopping trip, res-adm has more favorability for visiting and other trips, res-adm has more favorability for other trips, res-bus has more favorability for shopping and other trips, and peak hours has more choosing probability for shopping, recreational, and visiting trips than based trip aims. Individuals have less tendency to use personal vehicles and buses as trip modes to destinations with non-res and res-edu land use, respectively. Also, individuals have more tendency to use active transport as trip modes to destinations with res-adm and res-bus land use because these areas are more congested and travel distances are shorter than other areas [23]. The probability of selecting peak hours is low when individuals use their personal vehicles or active transport [1].

\section{Sensitivity analysis}

Formally, elasticity may be defined as a unitless measure that can describe the relationship between a percentage change of variables and some percentage change in the demanded quantity $[1,24]$. In the present study, disaggregate elasticities (for persons) were calculated for travel time, walking time, and travel distance which were continuous. Furthermore, the mean of disaggregate elasticities is aggregate elasticity [1]. Table 6 presents calculated elasticities.

These elasticities are interpretable. For example, a $1 \%$ increase in the travel time leads to an $2.03 \%$ decrease in the probability of choosing res-edu destination and departure time at traffic off-peak hours. Moreover, a $1 \%$ increase in walking time to the bus station leads to a $0.45 \%$ increase in the probability of choosing non-res destination and departure time at traffic peak hours.

\section{Conclusion}

The present study managed to examine the destination and departure time choices with interaction which was investigated by reflecting the correlation among unob- servable variables. While the multinomial logit was employed for destination choice, the binomial logit was used at day time. The related interaction was taken into account using three copulas including frank, AliMikhail-Haq copula (AMH), and products. The confirmation of the interdependency among these decisions obtained by the highly statically significant dependence parameters was one of the most significant findings of this study. These parameters were found to be stronger for the residential-educational and residential business destination. The joint model calibrated with a suitable goodness-of-fit and interpretable coefficients which demonstrated the effectiveness of the proposed model for joint modeling of these decisions. The main objective of this study was to calibrate the copulabased joint model for departure time and destination choices using the travel data of a developing country and to investigate aggregate elasticities as contributions. These models were employed to predict the future travel demands and describe the relationship among the dependent and independent variables. As a suggestion for future studies, segmenting data based on trip purposes is suggested. It also gives a better insight into utilizing land-use characteristics.

\section{References}

1. Seyedabrishami, S. and Izadi, A.R. "A copula-based joint model to capture the interaction between mode and departure time choices in urban trips", Transp. Res. Rec., 41, pp. 722-730 (2019).

2. Thorhauge, M., Cherchi, E., and Rich, J. "How flexible is flexible? Accounting for the effect of rescheduling possibilities in choice of departure time for work trips", Transp. Res. Part A Policy Pract., 86, pp. 177-193 (2016).

3. Sultana, S., Mode and Departure Time Choice Behavior of Non-Work Related Trips, Schulich School of Engineering (2019).

4. Sasic, A. and Habib, K.N. "Modelling departure time choices by a Heteroskedastic Generalized Logit (HetGenL) model: An investigation on home-based commuting trips in the Greater Toronto and Hamilton Area (GTHA)", Transp. Res. Part A Policy Pract., 50, pp. 15-32 (2013).

5. Hasnat, M.M., Faghih-Imani, A., Eluru, N., and Hasan, S. "Destination choice modeling using location- 
based social media data", J. Choice Model., 31, pp. 22-34 (2019).

6. Fox, J., Patruni, B., and Daly, A. "A new travel demand model for London: Estimation of the mode and destination choice models", Santa Monica, CA: RAND Corporation (2019). https://www.rand.org/pubs/ research_reports/RR2028.html

7. Clifton, K.J., Singleton, P.A., Muhs, C.D., and Schneider, R.J. "Development of destination choice models for pedestrian travel", Transp. Res. Part A Policy Pract., 94, pp. 255-265 (2016).

8. Hossain, S., Hasnine, M.S., and Habib, K.N. "A latent class joint mode and departure time choice model for the Greater Toronto and Hamilton Area", Transportation, 48, pp. 1-23 (2020).

9. Parsa, A.B., Kamal, K., Taghipour, H., and Mohammadian, A.K. "Does security of neighborhoods affect non-mandatory trips? A copula-based joint multinomial-ordinal model of mode and trip distance choices", TRB 98th Annual Meeting, Washington DC, Annual Meeting Compendium of Papers (No. 1903155) (2019).

10. Ding, C., Xie, B., Wang, Y., and Lin, Y. "Modeling the joint choice decisions on urban shopping destination and travel-to-shop mode: A comparative study of different structures", Hindawi Publishing Corporation, Discrete Dyn. Nat. Soc., 2014, Article ID: 492307, 10 pages (2014).

11. Bhat, C.R. "Analysis of travel mode and departure time choice for urban shopping trips", Transport. Res. B-Meth., 32(6), pp. 361-371 (1998).

12. Elmorssy, M. and Onur, T. "Modelling departure time, destination and travel mode choices by using generalized nested logit model: Discretionary trips", Int. J. Eng., 33(2), pp. 186-197 (2020).

13. Rasaizadi, A. and Kermanshah, M. "Mode choice and number of non-work stops during the commute: Application of a copula-based joint model", Sci. Iran., 25(3), pp. 1039-1047 (2018).

14. Seyedabrishami, S., Izadi, A.R., Rayaprolu, H.S., and Moeckel, R. "Car ownership: A joint model for number of cars and fuel types", Transp. Res. Proc., 41, pp. 572-576 (2019).

15. Ermagun, A., Rashidi, T.H., Arian, A., and Samimi, A. "Mode choice and escort decisions in school trips: Application of a copula-based model", TRB 93rd, Washington DC, Annual Meeting Compendium of Papers (No. 14-2560) (2014).

16. Bhat, C.R. and Sener, I.N. "A copula-based closedform binary logit choice model for accommodating spatial correlation across observational units", J. Geogr. Syst., 11(3), pp. 243-272 (2009).

17. Ben-Akiva, M.E., Lerman, S.R., and Lerman, S.R., Discrete Choice Analysis: Theory and Application to Travel Demand, MIT press (1985).
18. Portoghese, A., Spissu, E., Bhat, C.R., Eluru, N., and Meloni, I. "A copula-based joint model of commute mode choice and number of non-work stops during the commute", Int. J. Transp. Econ., 38, pp. 337-362 (2011).

19. Trivedi, P.K. and Zimmer, D.M. "Copula modeling: an introduction for practitioners", Found. Trends Econ., 1(1), pp. 1-111 (2007).

20. Liu, X.D., Pan, F., Cai, W.L., and Peng, R. "Correlation and risk measurement modeling: A Markovswitching mixed Clayton copula approach", Reliab. Eng. Syst. Saf., 19(7), p. 106808 (2020).

21. Wang, K., Bhowmik, T., Yasmin, S., Zhao, S., Eluru, N., and Jackson, E. "Multivariate copula temporal modeling of intersection crash consequence metrics: a joint estimation of injury severity, crash type, vehicle damage and driver error", Accid. Ana. Prev., 125, pp. 188-197 (2019).

22. Rasaizadi, A. and Askari, M. "Effect of family structure on urban areas modal split by using the life cycle concept", Int. J. Hum. Capital Urban Manage., 5(2), pp. 165-174 (2020).

23. Qi, Z., Lim, S., and Hossein Rashidi, T. "Assessment of transport equity to Central Business District (CBD) in Sydney, Australia", Transport. Lettr., 12(4), pp. 246256 (2020).

24. Hensher, D.A., Rose, J.M., and Greene, W.H., Applied Choice Analysis: A Primer, Cambridge University Press (2005).

\section{Biographies}

Arash Rasaizadi obtained his BS degree in Civil Engineering from Shahid Bahonar University of Kerman, Kerman, Iran in 2013. He also received his MS degree in Transportation Engineering from Sharif University of Technology (SUT), Tehran, Iran in 2015. He is currently a $\mathrm{PhD}$ candidate in the Transportation Engineering in Tarbiat Modares University (TMU), Tehran, Iran. His research interests include forecast and management of urban travel demand, short-term prediction of traffic parameters, and big data analysis.

Seyedehsan Seyedabrishami received his BS degree in Civil Engineering and $\mathrm{MS}$ and $\mathrm{PhD}$ degrees in Transportation Engineering from Sharif University of Technology (SUT) in 2004, 2006, 2011, respectively. $\mathrm{He}$ is now an Assistant Professor in the Civil and Environmental Engineering Department at Tarbiat Modares University (TMU) in Tehran. He has recently received a Georg Forster Fellowship as an experienced researcher from the Alexander von Humboldt Foundation in Germany. Since June 2017, he has been a visiting professor at the Research Group on Modeling Spatial Mobility at the Technical University of Munich (TUM). 